\title{
MEASUREMENT OF THE BRANCHING FRACTION FOR THE CABIBBO-
}

$$
\text { SUPPRESSED DECAY } \tau^{-} \rightarrow K^{-} \nu_{\tau} \text { * }
$$

C. A. B tocker, ${ }^{(a)} G, S$. Abrams, M. S. Alam ${ }^{(b)} A, B$ B ondel ${ }^{(c)}$

A. M. Boyarski, M. Breidenbach, D. L. Burke, W. C. Carithers,

W. Chinowsky, M. W. Coles, (d)S. Cooper(d) W. E. Dieterle,

J. B. Dillon, J. M. Dorfan, J. Dorenbosch, (e)M. W. Eaton,

G. J. Feldman, M. E, B, Franklin, G, GidaT, G. Goldhaber,

G. Hanson, K. G. Hayes, (e) T. Himeile) D. G. Hitlin, (f)

R. J. Hollebeek, W. R. Innes, J. A. Jaros, P. Jenni, (e)

A. D. Johnson, J. A. Kadyk, A. J. Lankford, R. R. Larsen,

M. Levi,(a) V. Lüth, R. E. Millikan, M. E. Neison,

C. Y. Pang, J. F. Patrick, M. L. Perl, B. Richter,

A. Roussarie, D. L. Scharre, R. H. Schindler, (e)

R. F. Schwitters, (a) J. L. Siegrist, (e) J. Strait,

H. Taureg,(e) M. Tonutti, (g) G. H. Trilling, E. N. Vella,

R. A. Vidal, I. Videau, (c) J. M. Weiss, and H. Zaccone.(h)

Stanford Linear Accelerator Center

Stanford University, Stanford, California 94305

and

Lawrence Berkeley Laboratory and Department of Physics

University of California, Berkeley, California 94720

\section{ABSTRACT}

The branching fraction for the Cabibbo-suppressed decay $\tau^{-} \rightarrow K^{-} \nu_{\tau}$ is measured from data obtained with the Mark II detector at SPEAR. Fifteen events containing a $K_{-}^{ \pm}$in coincidence with a muon or electron of opposite charge are observed. It is determined that $\mathrm{B}\left(\tau^{-} \rightarrow \mathrm{K}^{-} \nu_{\tau}\right)$ $=(1.3 \pm 0.5) \%$.

\section{Submitted to Physical Review Letters}

* Work supported in part by the Department of Energy, contracts DE-AC03-76SF00515 (SLAC) and W-7405-ENG-48 (LBL).

(a) Harvard University, Cambridge, MA 02138

(b) Vanderbjit University, Nashvilie, TN 37235

(c) LPNHE, Ecole Polytechnique, Palaiseau, France

(d) DESY, Hamburg, Fed. Rep. of Germany

(e) EP Division, CERN, Geneva, Switzerland

(f) California Institute of Technology, Pasadena, CA 91125

(g) Universität Bonn, Fed. Rep. of Germany

(h) CEN-Saclay, France 
We'present a measurement of the branching fraction for the decay (1) $\tau^{-} \rightarrow K^{-} \nu_{\tau}$, the Cabibbo-suppressed counterpart of the decay $\tau^{-} \rightarrow \pi^{-} \nu_{\tau}$. The presence of this decay indicates that the Cabibbo-suppressed axial-vector hadronic current is coupled to the $\tau$ and supports the hypothesis that the standard weak current mediates decays of the $\tau$. Previously we published $(2,3)$ measurements of the branching fractions for $\tau^{-} \rightarrow \pi^{-} \nu_{\tau}, \tau^{-} \rightarrow \rho^{-} \nu_{\tau}$, and $\tau^{-} \rightarrow K^{*}(892) \nu_{\tau}$, a 11 in good agreement with theoretical expectations based on a standard hadronic weak coupling. These measurements indicate that the Cabibbo-favored axial-vector and vector hadronic currents and the Cabibbo-suppressed vector hadronic current, respective$1 y$, are coupled to the $\tau$ with the expected strength.

The measurement is based on Mark II SPEAR data over the center-of-mass energy range $3.88<\mathrm{E}_{\mathrm{cm}}<6.7 \mathrm{GeV}$, with a total integrated luminosity of $17,000 \mathrm{nb}^{-1}$, corresponding to 47,000 produced $\tau^{+}{ }_{\tau}^{-}$pairs. All aspects of the Mark II solenoidal detector pertinent to this measurement have been fully discussed elsewhere. (4)

The decay $\tau^{-} \rightarrow \mathrm{K}^{-} \nu_{\tau}$ is identified by the topology

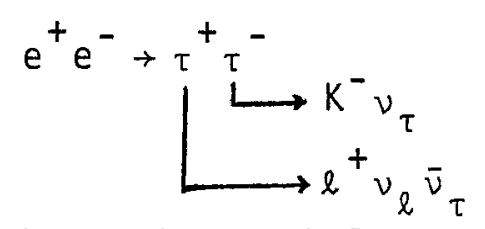

which results in two charged particles and no photons in the detector. The symbol \& represents either an electron or a muon. Events are required to have exactly two, oppositely 
charged'particles and no photons with energy above $100 \mathrm{MeV}$. The two charged particles are required to be acoplanar(5) by at least $20^{\circ}$ and to form a good vertex within $10 \mathrm{~cm}$ of the beam interaction point, as measured along the beam direction. One of the charged particles is required to be a lepton and the other not to be identified as a lepton. The selection criteria for leptons are identical to those used in the analysis of $\tau^{-} \rightarrow \pi^{-} v_{\tau}$ and are discussed in Ref. 2.

The mass-squared of the non-lepton track is calculated from the measured time-of-flight (TOF), momentum, and path length. Figure 1 shows a scatterplot of mass-squared versus momentum. The contours are three standard deviation limits (calculated using a 310 psec TOF resolution and average path length) for electrons and pions and two standard deviation limits for kaons and protons. A clear kaon signal is seen. All particles in the proton region of fig. 1 are consistent with coming from either beam-gas interactions or tails of the $e, \mu, \pi$, and $k$ distributions. The absence of kaons in 2-prong lepton events with a vertex displaced along the beam direction demonstrates that beam-gas interactions are not a background to lepton - K events.

Kaon candidates have a measured TOF within 2.5 standard deviations of the expected time (calculated from the momentum, path length, and mass) for a kaon, greater than 4 standard deviations from the expected time for a pion, and greater than 2 standard deviations from the expected time for a proton. 
The TOF resolution function including non-Gaussian tails is measured using two-prong events with either a low-momentum pion $(100<p<125 \mathrm{MeV} / \mathrm{c})$ or two muons with the full beam energy (u-pair events). In Fig. 2, the difference between the measured and expected TOF is shown for a typical sample of u-pair events. The curve is a Gaussian fit to the data. The $\mu$-pair events and low-momentum pions give similar results, and the averages are summarized in Table I for various blocks of data.

Fifteen $\& K$ events satisfy the selection criteriadescribed above. From the observed number of $\ell(e, \mu, \pi)$ events in Fig. 1 and from Table I, it is estimated that $1.9 \pm 0.5$ $\ell(\pi, \mu, e)$ events are classified as $\ell K$ events. From a Monte Carlo program, it is calculated (based on $B\left(\tau^{-} \rightarrow \mathrm{K}^{-}(892) \nu_{\tau}\right)$ $=(1.7 \pm 0.7) \%$ from Ref. 3) that the process $e^{+} e^{-} \rightarrow_{\tau_{\tau}^{+}}^{+}$ $\rightarrow\left(e^{+} \nu_{\ell} \bar{\nu}_{\tau}\right)\left(K *^{-} \nu_{\tau}\right) \rightarrow(\ell \nu \nu)\left(K^{-} \pi^{0} \nu_{\tau}\right)$ contributes $2.5 \pm 1.0$ events to the $\ell K c l a s s$. To insure that the lepton tags are not misidentified hardons, events are selected with the same criteria as $\ell k$ events except the tagging particle is required not to be a lepton, resulting in $30 \mathrm{KX}$ events. Ascribing al1 the 15 ek events to misidentification of the lepton tag implies a $33 \%$ misidentification probability, inconsistent with the measured values of $\$ 5 \%$ for the Mark II. In fact, the ratio of $K X$ to $\& K$ events is consistent with both classes of events coming solely from $\tau$ production and decay. Classification of $\ell K(K X)$ events as $K X(\ell K)$ events is included in 
the efficiency calculation. Subtracting the background events gives a net signal of $10.6 \pm 3.9 \pm 1.1$ events. (6) The statistical significance of this result can be judged from the fact that the probability that 4.4 background events fluctuate (including the uncertainty in the background calculation) to give 15 or more events is $5 \times 10^{-4}$. Of the 15 eK events, $11(4)$ have an electron (muon)tag, consistent with the relative electron/muon tagging efficiency of $\sim 1.8$. The momentum spectra for the lepton tags and the $K$ 's are well reproduced by the Monte Carlo program. In particular, the lepton momentum spectrum is "hard", that is, 8 of the 15 leptons have momenta above $700 \mathrm{MeV} / \mathrm{c}$.

Since charm particle decays produce both leptons and strange particles, it is important to establish that the ek events are not from charm production. Since there are no $\ell \pm K \pm$ events, it can be assumed that most of the $\ell \pm K \mp$ events have no undetected charged particles. The relatively high charged and neutral multiplicities of charm events coupled with the good solid angle coverage of the Mark II detector make it unlikely that charm events populate the low multiplicity $\ell-K-$ no photon topology. Semileptonic charm decays produce leptons with the same sign of the charge as the charm quark. The decays $D^{ \pm}, \Lambda_{C} \pm \rightarrow K^{+}+$neutrals are forbidden by the $\Delta S=\Delta C$ rule and are not background problems. A possible source of background is $e^{+} e^{-} \rightarrow D^{0} \bar{D}^{0}+n_{\pi}^{0}$ where $D^{0} \rightarrow \ell^{+} K^{-}$. + neutrals and $\bar{D}^{\circ} \rightarrow$ all neutrals. A Monte Carlo simulation 
program; adjusted to agree with the observed properties of $D$ decays has been used to estimate that at most 0.1 of the lK events are from $D$ production. The $F$ meson can decay via two distinct diagrams - the charm quark can decay $(F=c \bar{s} \rightarrow$ $\bar{s} s W \rightarrow \bar{s} s+e v, \mu v, u \bar{d})$ or the charm and strange quark can annihilate $(c \bar{s} \rightarrow W \rightarrow e v, \mu \nu, \tau \nu, u \bar{d})$. The decay diagram gives many kaons but few high momentum leptons. The annihilation diagram gives high momentum electrons but few kaons. Using the branching fractions from Quigg and Rosner(7) and assuming a conservative upper limit on $F \bar{F}$ production of $30 \%$ of the $\mu$-pair cross section, it is estimated that at most 0.2 lK events come from $F$ production for any combination of the decay and annihilation diagrams. Due to the expected sma11 rates of Cabibbo-suppressed charm decays, they are a negligible background. Finally, the leptons from charm production have a "softer" momentum spectrum (almost all of these leptons would have momenta below $700 \mathrm{MeV} / \mathrm{c}$ ) than that observed in the $\ell k$ events.

The detection efficiency for the ek events, calculated from a Monte Carlo program, is $2.4 \%$ assuming e- $\mu$ universality. Combining this efficiency, the number of produced $\tau^{+} \tau^{-}$pairs, and the number of signal events gives

$$
B\left(\tau^{-} \rightarrow K^{-} \nu_{\tau}\right) \cdot B\left(\tau^{-} \rightarrow e^{-} \bar{\nu}_{\ell} \nu_{\tau}\right)=(2.3 \pm 0.8 \pm 0.3) \times 10^{-3} \text {. }
$$

Using the Mark I measurement (2) of

$$
B\left(\tau^{-} \rightarrow \ell^{-} \bar{v}_{\ell} \nu_{\tau}\right)=(17.6 \pm 1.2) \%
$$


yields

$$
B\left(\tau^{-} \rightarrow K^{-} \nu_{\tau}\right)=(1.3 \pm 0.5) \% .
$$

The quoted error consists of the statistical uncertainty plus systematic uncertainties of $13 \%$ for the background subtraction, $5 \%$ for lepton misidentification, $6 \%$ for luminosity, and $10 \%$ for the Monte Carlo efficiency. The data are corrected for initial state radiation effects. Repeating the analysis by requiring that the TOF for kaon candidates be from 3.0 to 4.5 standard deviations from the TOF expected for pions gives $B\left(\tau^{-} \rightarrow K^{-} \nu_{\tau}\right)=1.3$ to $1.4 \%$, indicating that the TOF distribution, particularly the non-Gaussian tajls, has been handled properly.

The theoretical expectation for the $\tau^{-} \rightarrow K^{-}{ }_{\tau}$ branching fraction can be calculated from either the $\tau^{-} \rightarrow \pi^{-} \nu_{\tau}$ or $\tau^{-} \rightarrow e^{-} \bar{\nu}_{e} \nu_{\tau}$ branching fractions. From the calculations of Tsai, (8)

$$
B\left(\tau^{-} \rightarrow K^{-} \nu_{\tau}\right)=\frac{f_{K}{ }^{2}}{f_{\pi}^{2}} \frac{\left(1-\frac{M_{K}{ }^{2}}{M_{\tau}{ }^{2}}\right)^{2}}{\left(1-\frac{M_{\pi}{ }^{2}}{{ }^{2}}\right)^{2}} B\left(\tau^{-} \rightarrow \pi^{-} \nu_{\tau}\right)
$$

and

$$
B\left(\tau^{-} \rightarrow K^{-} \nu_{\tau}\right)=\frac{12 \pi^{3} f_{K}{ }^{2}}{M_{\tau}{ }^{2}}\left(1-\frac{M_{K}{ }^{2}}{M^{2}}\right)^{2} B\left(\tau^{-}+e^{-} \bar{\nu}_{e} \nu_{\tau}\right),
$$

where $f_{\pi}$ and $f_{K}$ are constants obtained from the pion and kaon lifetimes, respectively. Using the Mark II measurements (2) of $B\left(\tau^{-} \rightarrow \pi^{-} \nu_{\tau}\right)=(11.7 \pm 1.8) \%$ and $B\left(\tau^{-} \rightarrow e^{-} \bar{v}_{e} \nu_{\tau}\right)=(17.6 \pm 1.2) \%$ gives estimates of $(0.74 \pm 0.11) \%$ and $(0.63 \pm 0.04) \%$, respectively, 
for $B\left(\tau^{r} \rightarrow K^{-} \nu_{\tau}\right)$, in agreement with the experimental value presented in this letter. This result is also consistent with the upper limit of $\mathrm{B}\left(\mathrm{\tau}^{-} \rightarrow \mathrm{K}^{-} \nu_{\tau}\right)<1.6 \%$ given by the DASP collaboration.

In summary, the decay $\tau^{-} \rightarrow K^{-} \nu_{\tau}$ has been observed at the level expected if the $\tau$ couples to the standard cabibbosuppressed axial-vector hadronic weak current.

Table I

TOF Performance for Various Data Samples

\begin{tabular}{|c|c|c|c|}
\hline $\begin{array}{c}\mathrm{E}_{\mathrm{cm}} \\
(\mathrm{GeV})\end{array}$ & $\begin{array}{l}\text { Number of } \\
\text { Produced } \tau \text { Pairs }\end{array}$ & $\begin{array}{c}\text { TOF } \\
(p s e c)\end{array}$ & $\begin{array}{l}\text { Measured Probability } \\
\text { that TOF-t exp }>4 \sigma \\
(x 10-3) \text { exp }\end{array}$ \\
\hline $3.88-4.5$ & 11,100 & 315 & $2.3 \pm 0.9$ \\
\hline $4.5-6.0$ & 11,100 & 283 & $0.2 \pm 0.2$ \\
\hline 5.2 & 15,300 & 320 & $0.7 \pm 0.4$ \\
\hline $6.0-6.7$ & 9,500 & 310 & $1.2 \pm 0.8$ \\
\hline
\end{tabular}

This work was supported in part by the Department of Energy, Under contracts DE-AC03-76SF00515 (SLAC) and W-7405-ENG-48 ( $L B L)$. Support for individuals came from the listed institutions.

\section{References}

1. All reactions in this paper also imply the charge conjugate reaction. Thus, $\tau^{-} \rightarrow K^{-} v_{\tau}$ stands for itself and for $\tau+K^{+} \bar{\nu}_{\tau}$.

2. C. A. B 1ocker et al., SLAC-PUB-2820 and to be published in Phys. Lett. B. 
3. G. 'S. Abrams et a 1., Phys. Rev. Lett. 43, 1555 (1979). J. M. Dorfan etal., Phys. Rev. Lett. 46, 215 (1981).

4. G. S. Abrams et a 1. Phys. Rev. Lett. 43, 477 (1979.

5. The acoplanarity angle is the angle between the plane containing the beam and one final state particle and the plane containing the beam and the other final state particle.

6. The first error is statistical, the second is systematic.

7. Quigg and Rosner, Phys. Rev. D17, 239 (1978).

8. Y. S. Tsai, Phys. Rev. D4, 2821 (1971).

9. S. Yamada, Proc. of the 1977 International Symposium on Lepton and Photon Reactions at High Energies, Hamburg, p. $-69(1977)$.

\section{Figure Captions}

1. Scatterplot of TOF mass-squared versus momentum for acoplanar, two-prong events with a lepton tag. The contours are three standard deviation limits for electrons and pions and two standard deviation limits for kaons and protons.

2. Measured minus expected TOF for $\mu$-pair events from the 5.2 GeV data sample. 


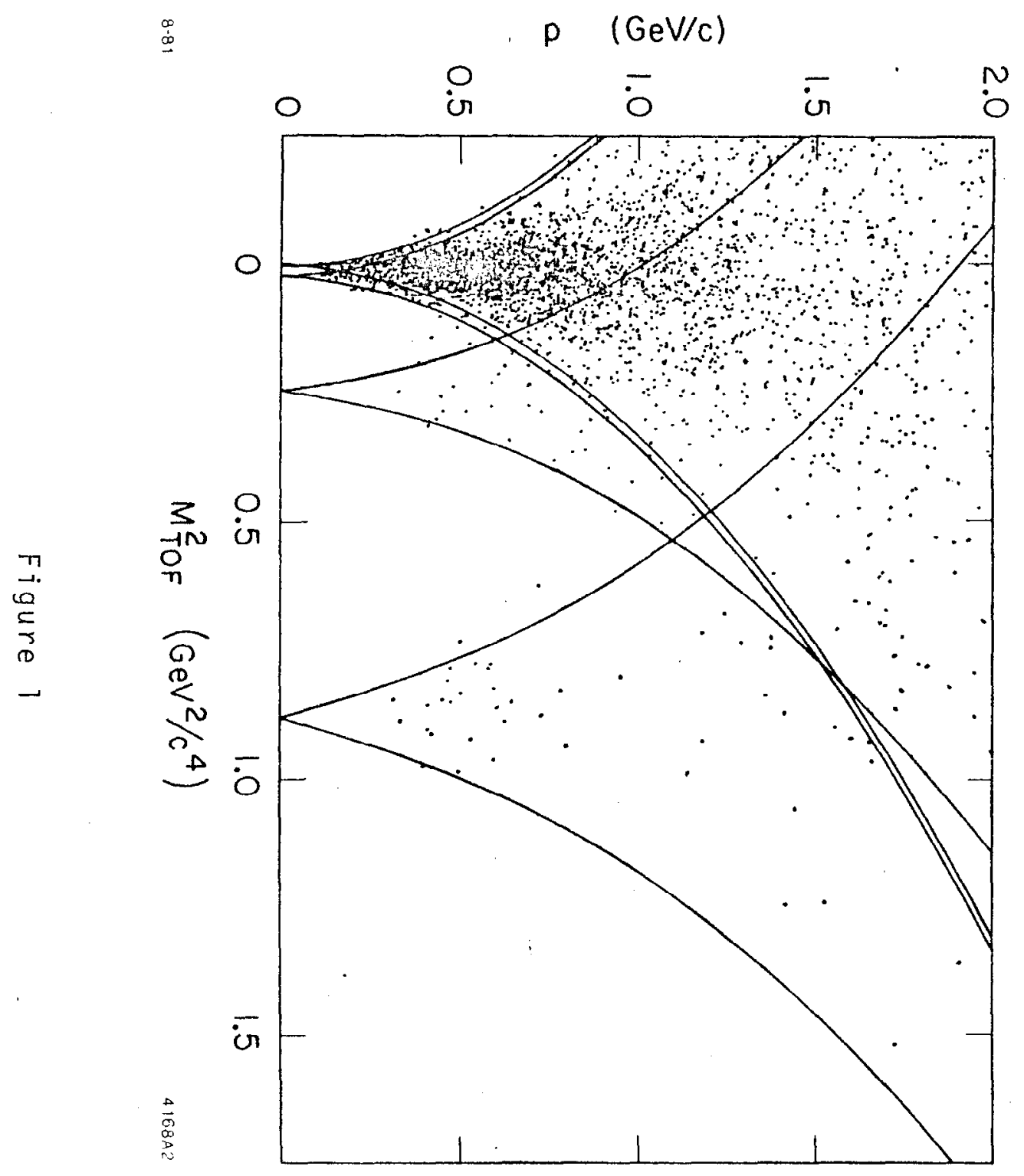




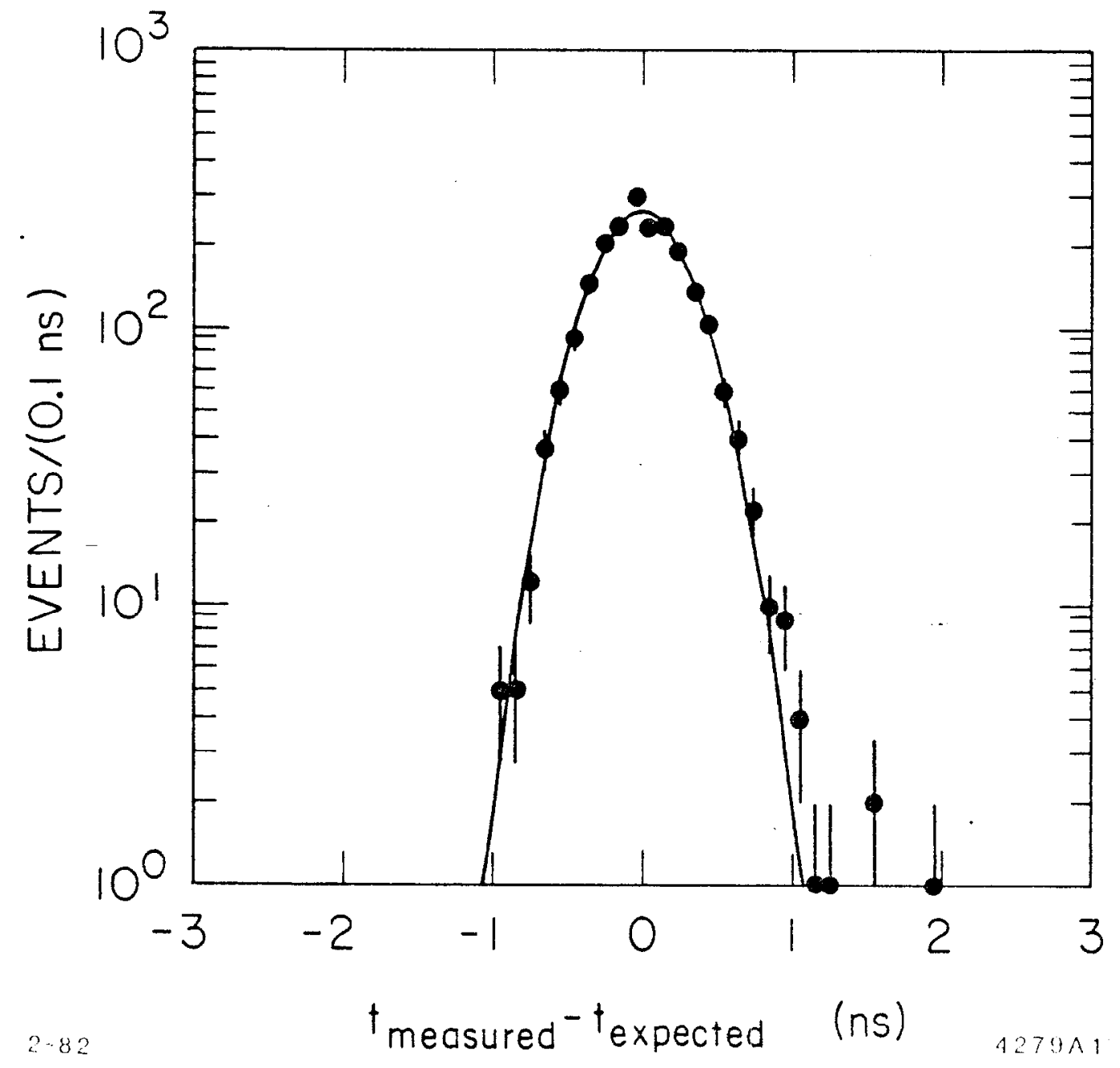

Figure 2 